\section{Multiple spider telangiectasias in a breast cancer patient on T-DM1 treatment*}

\section{DOI: http:/ /dx.doi.org/10.1590/abd1806-4841.20187968}

Juncal Ruiz-Rivero ${ }^{1}$

Celia Horcajada-Reales ${ }^{1}$

Juan C. Tardío ${ }^{2}$

Jesús Manuel Borbujo-Martínez ${ }^{1}$

Dear Editor,

Human epidermal growth factor receptor 2 (HER2)-positive breast cancer $(B C)$ are aggressive tumours with HER2 gene amplification and HER2 protein overexpression. ${ }^{1,2}$ The distinction between HER2-positive and Her2-negative carcinomas is of practical importance since the development of anti-HER2 agents has improved both recurrent rates and disease-free intervals. Up to date, there are four targeted therapies especially developed against HER2-positive BC: trastuzumab, pertuzumab, lapatinib and T-DM1 (or ado-trastuzumab emtansine). Of all four, T-DM1 was the last one to be added to the therapeutic arsenal, which means that its short/medium and long-term side effects, including the cutaneous events, are yet to be established.

We present a 56-year-old woman with history of stage IV HER2-positive BC that was referred to our outpatient clinic. She had been first diagnosed ten years before and treated initially with surgery and radiotherapy but progressed on multiple lines of chemotherapy (including paclitaxel, trastuzumab and pertuzumab) due to two relapses and disease progression. At the time of consultation, she had been under T-DM1 treatment $(3.6 \mathrm{mg} / \mathrm{kg} / \mathrm{IV}$ every 3 weeks) for 1 year. She had tolerated well the medication except for some self-limited nasal and gum bleeding. She referred eight months of asymptomatic skin lesions over her trunk and proximal extremities. Physical examination showed well defined vascular-like plaques of less than $5 \mathrm{~mm}$ size (Figure 1). Dermoscopy images of the lesions exhibited a central red spot connected to peripheral telangiectatic

Received 13 December 2017.

Accepted 26 March 2018.

* Work conducted at the Hospital Universitari Sagrat Cor, Barcelona, Spain.

Financial support: None.

Conflict of interest: None.

Department of Clinical and Surgical Dermatology and Venereology, Hospital Universitario de Fuenlabrada, Madrid, Spain.

2 Department of Pathological Anatomy, Hospital Universitario de Fuenlabrada, Madrid, Spain.

MAILING AdDRESS:

Juncal Ruiz-Rivero

E-mail: juncal.ruiz@salud.madrid.org

(C2018 by Anais Brasileiros de Dermatologia vessels with a swirling appearance (Figure 2). Biopsy revealed some branching small blood vessels in the superficial dermis, which were lined by a prominent endothelium (Figure 3). Laboratory findings

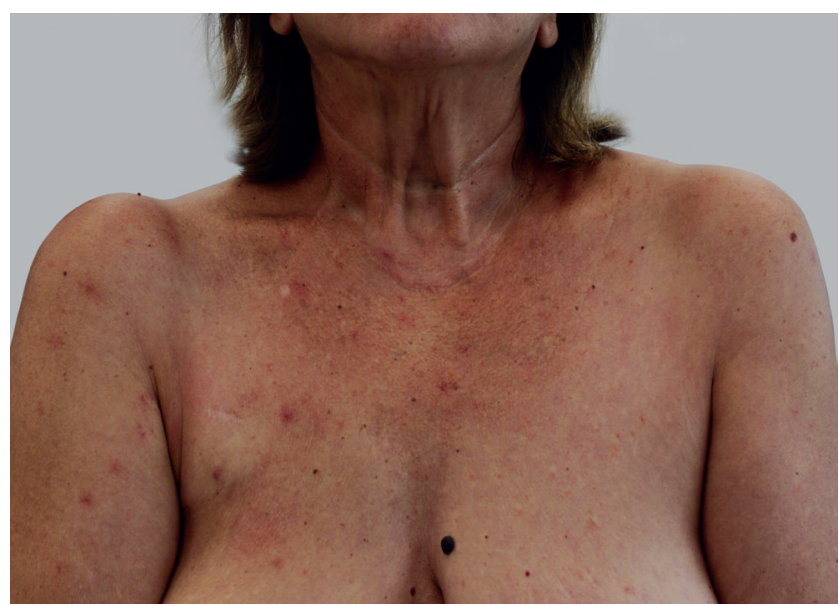

Figure 1: Multiple spider nevi over the trunk and proximal extremities



Figure 2: Dermoscopy image of one of the lesions, showing expanded branching vessels



FIGURE 3: Branching small blood vessels lined by a prominent endothelium. (Hematoxylin \& eosin, x20) 
revealed mild elevation in liver enzymes levels. Red and white blood cell and platelet counts had no alterations and all biochemical parameters were in the normal range. Based on these data, we made the diagnosis of spider nevi secondary to T-DMI treatment. The lesions did not require specific treatment so the patient was instructed on general skin care.

T-DM1 is a novel antibody-drug conjugate that combines the antitumor properties of trastuzumab against HER2 with the cytotoxic activity of emtansine, a microtubule-inhibitory agent. T-DMI was first approved by the FDA after results of the EMILIA, a randomized international phase III clinical trial which compared T-DMI monotherapy versus lapatinib and capecitabine in 991 women with HER2-positive advanced or metastatic BC who had been previously treated with trastuzumab and a taxane. T-DM1 showed better progression-free survival as well as better overall survival and safety. In addition, it was better tolerated. The main adverse events reported were fatigue, nausea, diarrhoea, elevated transaminases, anaemia and thrombocytopenia with secondary haemorrhage. Thrombocytopenia and elevated serum concentrations of liver enzymes were the most commonly reported grade 3 or 4 events. ${ }^{3}$

The only data on cutaneous side effects are described in oncological journals and consist of mucositis and hand foot syndromes. Recently, cutaneous and mucosal telangiectasias have also been described. ${ }^{4}$ Sibaud et al described the first series of mucocutaneous telangiectasia in five women under T-DM1 treatment in 2014. ${ }^{4}$ All of them developed grade I transaminitis and three referred history of mild/limited gingival bleeding and/or epistaxis. Thrombocytopenia from grade 1 to 3 was only registered in two patients. Asymptomatic telangiectasias were developed between 2.5 and 14 months after T-DM1 was initiated. Since transaminitis is one of the major side effects of T-DM1 and spider nevi are also seen in other hepatic diseases, they postulate that liver injury may play a role on its development. They also try to relate the mechanism of development of telangiectasia in hereditary hemorrhagic telangiectasia (HHT) by disruption of cytoskeletal microtubules with the cytotoxic activity of emtansine.

In addition, a case of skin telangiectasias and pulmonary arterial hypertension (PAH) was described by Kwon et al a year later. ${ }^{5}$ In their article, they propose that some dysfunction in ALK-1 (activin receptor-like kinase) may explain both mucocutaneous telangiectasia and PAH, since ALK-1 mutations have been found to be related to $\mathrm{PAH}$ in patients with $\mathrm{HHT}$.

Herein, we describe a new case of skin telangiectasia or spider nevi related to T-DM1 treatment. To our knowledge, this is the first description in the dermatological field. Since T-DM-1 is a recently introduced drug used in $\mathrm{BC}$ and whose use is expected to increase over the next years, we consider of great importance being aware of this potential cutaneous event. $\square$

\section{REFERENCES}

1. Tao, Shi A, Lu C, Song T, Zhang Z, Zhao J. Breast Cancer: Epidemiology and Etiology. Cell Biochem Biophys. 2015;72:333-8.

2. Makki J. Diversity of Breast Carcinoma: Histological Subtypes and Clinical Relevance. Clin Med Insights Pathol. 2015;8:23-31.

3. Verma S, Miles D, Gianni L, Krop IE, Welslau M, Baselga J, et al. Trastuzumab emtansine for HER2-positive advanced breast cancer. N Engl J Med. 2012;367:1783-91.

4. Sibaud V, Niec RE, Schindler K, Busam KJ, Roché H, Modi S, et al. Adotrastuzumab emtansine-associated telangiectasias in metastatic breast cancer: a case series. Brest Cancer Res Treat. 2014;146:451-6.

5. Kwon Y, Gomberg-Maitland M, Pritzker M, Thenappan T. Telangiectasia and Pulmonary Arterial Hypertension following Treatment with Trastuzumab Emtansine. A case report. Chest. 2016;149:e103-5.

\section{AUTHORS CONTRIBUTIONS}

Juncal Ruiz-Rivero $\quad$ (iD) ORCID 0000-0002-7068-9056

Elaboration and writing of the manuscript, Obtaining, analyzing and interpreting the data, Critical review of the literature, Critical review of the manuscript

Celia Horcajada-Reales iD ORCID 0000-0003-0333-2134

Approval of the final version of the manuscript, Critical review of the literature, Critical review of the manuscript

Juan C. Tardío ORCID 0000-0003-1805-961X Conception and planning of the study, Obtaining, analyzing and interpreting the data Jesús Manuel Borbujo-Martínez iD ORCID 0000-0003-1922-4699 Conception and planning of the study, Effective participation in research orientation, Critical review of the manuscript

How to cite this article: Ruiz-Rivero J, Horcajada-Reales C, Tardio JC, Borbujo-Martínez JM. Multiple spider telangiectasias in a breast cancer patient on T-DM1 treatment. An Bras Dermatol. 2018;93(6):938-9. 